\title{
Building up the Quality process
}

\author{
Alan Lawrence \\ Editor
}

0 $\mathrm{n}$ behalf of my associate editors, commentators and production team, I would like to say a very heartfelt thank you to our readers for the positive response to the first edition. Evidence-Based Dentistry is clearly meeting a need. We are continuously refining the editorial process in the light of experience and feedback to ensure that the supplement continues to meet those needs. I would also like thank all those of you who wrote to offer your services as future commentators. This is greatly appreciated.

We believe it is essential that EvidenceBased Dentistry maintains a consistently high standard. It may be helpful to describe the main steps of the process so that readers understand the quality standards, filters and systems that we are now using. These are similar to those used in the Journal of Evidence-based Medicine. Essentially there are 5 steps to the process.

The first part of the process is to look, worldwide, for relevant clinically useful published papers. Such research papers may confirm present practice, indicate where changes are required, or point out and clarify the need for further research. Most importantly they contribute to the evidence base behind modern clinical practice.

We look, first of all, at journals that publish a high percentage of evidencebased papers. ${ }^{1}$ We are also very keen to know of any relevant topical and important papers published in any other peer reviewed journals. For this we are developing a team of spotters in each specialty in dentistry to look regularly for, and alert us about, primary papers which could be summarised in the supplement. Please contact us if you could act as a spotter for a journal in your own specialty.

When we identify, or are notified about, possible papers we begin the second step. This is the critical appraisal. Critical appraisal uses systems developed by the evidence based medicine

\section{The ultimate test is whether practice is changing in response to better evidence. This should actually improve the health of our patients. Evidence-based dentistry is a practical discipline and must not be confined to ivory towers.}

group led by Professor David Sackett and the Critical Appraisal Skills Programme in Oxford, ${ }^{2}$ and examines the scientific validity and clinical importance of the original paper.

If the paper passes the critical appraisal phase, the third stage is when we prepare an expanded factual abstract based on the original paper. This includes statistical information on numbers needed to treat, sensitivity, and specificity where this would be helpful for readers.

The fourth step is to invite an independent commentator who has clinical and research experience in the relevant clinical areas and related fields to write a commentary. The commentator is not there to provide a second peer review of the original paper, but is expected to provide a brief expert summary of the context of an article, any key methodological issues and the clinical applications that the study's findings warrant.

Fifthly the combined summary and commentary are introduced by a brief 'headline' that captures the take-home message, and the summary and the commentary are sent to the senior author of the original paper for any comment and clarification.

Whilst the summaries form a major part of Evidence-Based Dentistry we feel that articles on developing or practising evidence based dentistry are also important for readers. We will be aiming to tell readers about useful sources of information and help, especially where these are easily available on the web. Forthcoming issues will explore some of these sources and aids. We have grouped all these articles in the toolbox section.

We would welcome original articles related to the practice and practicalities of the evidence-based approach and have produced guidelines for authors on the inside back cover. All articles are peer reviewed and I would like to thank our peer reviewers who are important members of the quality process.

Finally it is vital that you, the reader, tell us what you think. The evidence based approach enables practitioners to respond effectively to the needs and queries of patients. The ultimate test is whether practice is changing in response to better evidence. This should actually improve the health of our patients. Evidence-based dentistry is a practical discipline and must not be confined to ivory towers.

1 Richards D. Which journals should you read to keep up to date? Evidence-Based Dentistry 1998 November 1: 22-25

2 http://www.ihs.ox.ac.uk/casp/ 